\title{
Perfil sociodemográfico y apoyo social percibido de personas mayores en condiciones de vulnerabilidad
}

\author{
Sociodemographic profile and perceived \\ social support of vulnerable older adults
}

Perfil sociodemográfico e percepção de suporte social de idosos em condições de vulnerabilidade

\author{
María Julia Xifra'
}

Recibido: 15 de octubre 2020 • Enviado para modificación: 18 de noviembre $2020 \bullet$ Aceptado: 13 de febrero 2021

Xifra, M.J. (2020). Perfil sociodemográfico y apoyo social percibido de personas mayores en condiciones de vulnerabilidad. Revista Ocupación Humana, 20(2), 10-24. https://doi.org/10.25214/25907816.996

\section{RESUMEN}

El propósito del estudio fue describir el apoyo social percibido y las variables sociodemográficas en personas mayores de la ciudad de Mar del Plata, Argentina, que concurren a comedores comunitarios o reciben bolsón de alimentos. Desde un enfoque metodológico cuantitativo, se llevó a cabo un estudio descriptivo de corte transversal. Para la recolección de datos se utilizaron un cuestionario sociodemográfico y el Cuestionario de Apoyo Social de Sherbourne y Stewart. Entre las características sociodemográficas prevalentes se destacan el bajo nivel de instrucción y el elevado porcentaje de personas viudas y que viven en soledad. Los puntajes obtenidos en el total del Cuestionario de Apoyo Social y sus dimensiones mostraron valores medios respecto a los puntos de corte propuestos. Se concluye que, aunque la cantidad de vínculos de que disponen las personas mayores no es escasa ni se evidencian disrupciones severas, coexisten la menor disponibilidad de apoyos instrumental y emocional informacional, un porcentaje alto de personas mayores que viven en soledad y una escasa participación en centros de jubilados. Desde la Terapia Ocupacional comunitaria se aspira a prestar especial atención a estos grupos de personas mayores vulnerables, sus posibilidades de inclusión y participación.

\section{PALABRAS CLAVE}

envejecimiento, personas mayores, apoyo social, vulnerabilidad social, Terapia Ocupacional

'Licenciada en Terapia Ocupacional. Especialista en Docencia Universitaria. Magíster en Psicogerontología. Docente, Universidad Nacional de Mar del Plata. Mar del Plata, Argentina. julyxifra@yahoo.com.ar iD https://orcid.org/0000-0001-9313-8612 


\begin{abstract}
The main goal of this work was to describe the perceived social support and sociodemographic variables of older people who attend community dining rooms or receive food packages in Mar del Plata, Argentina. It is a descriptive study with a quantitative approach. The information was collected through a sociodemographic questionnaire and the Sherbourne \& Stewart Social Support Questionnaire. The prevalent sociodemographic characteristics were a low educational level, a high percentage of widows and widowers and a large number of older adults who live alone. The total scores in the Social Support Questionnaire and its dimensions presented average values with respect to the proposed cut-off points. It is concluded that, although the number of relationship available to older people is not scarce and there is no evidence of severe disruptions, there is less instrumental and emotional informational support available, a high percentage of older people living alone, and a low participation in retirement centers. Community Occupational Therapy aims to pay special attention to these groups of vulnerable older people, their possibilities of inclusion and participation.
\end{abstract}

\title{
KEY WORDS
}

aging, older adults, social support, social vulnerability, Occupational Therapy

\section{RESUMO}

O objetivo do estudo foi descrever o apoio social percebido e as variáveis sociodemográficas em idosos na cidade de Mar del Plata, Argentina, que frequentam refeitórios comunitários ou recebem uma cesta básica de comida. A partir de uma abordagem metodológica quantitativa, realizou-se um estudo transversal descritivo. Para a coleta de dados, foram utilizados um questionário sociodemográfico e o Questionário de Apoio Social de Sherbourne e Stewart. Dentre as características sociodemográficas que prevalecem destacam-se a baixa escolaridade e o alto percentual de pessoas viúvas e que moram sozinhas. Os escores obtidos no total do Questionário de Apoio Social, e suas dimensões, apresentaram valores médios em relação aos pontos de corte propostos. Conclui-se que, embora o número de vínculos disponíveis para idosos não seja escasso e não haja rupturas graves, há menor disponibilidade de suporte informacional instrumental e emocional, bem como elevado percentual de idosos que moram sozinhos, além de uma baixa participação em centros de aposentadoria. A Terapia Ocupacional Comunitária visa dar atenção especial a esses grupos de idosos vulneráveis, as suas possibilidades de inclusão e à participação.

\section{PALAVRAS-CHAVE}

envelhecimento, idosos, apoio social, vulnerabilidade social, Terapia Ocupacional

\section{Introducción}

La población en el mundo está envejeciendo. La mayoría de los países están experimentando un incremento en el número y la proporción de personas mayores, por lo que el envejecimiento poblacional apunta a convertirse en una de las transformaciones sociales más significativas del siglo XXI. Este fenóme- 
no tendrá consecuencias macro y microsociales, por lo tanto, impactará en diversas áreas y niveles (Organización de las Naciones Unidas [ONU], 2019).

Se prevé que la población mundial mayor de 60 años se elevará de 900 a más de 1.400 millones de personas entre 2015 y 2030 (Huenchuan, 2018). En ese contexto, la preocupación no debe enfocarse solo en las cifras esperadas en este grupo poblacional, sino en el escenario en el que ocurre, caracterizado por la desigualdad, la pobreza, el agotamiento de un modelo de crecimiento económico insostenible, entre otros (Huenchuan, 2018).

La República Argentina exhibe una transición demográfica acelerada. Los últimos datos oficiales relevados en la ciudad de Mar del Plata, según el Censo Nacional (Instituto Nacional de Estadística y Censos [INDEC], 2010), arrojaron que el total de la población es de 618.989 personas, de las cuales 119.006 son mayores de 60 años (19,2\%). El análisis de datos publicados más recientemente por la Pontificia Universidad Católica Argentina permite observar un incremento del porcentaje de personas de este grupo etário en la ciudad, llegando al 25,8\% (Cicciari, 2017). En cuanto a la distribución por estrato socioeconómico, el 43,7\% pertenece al estrato muy bajo (Cicciari, 2017).

De acuerdo con Manes et al. (2016), la vejez con frecuencia se asocia con estereotipos, prejuicios e imaginarios negativos, y se la piensa como una realidad homogénea, regida por ciertas pautas culturales universalizadas. Estas concepciones, desde una connotación negativa, tienden a asociar a la persona mayor con abandono, soledad, discapacidad, marginalidad, deterioro y enfer- medad. Refieren estos autores que tales preconceptos no son ajenos a los criterios propios del capitalismo y de la cultura occidental y crean subjetividades que impactan en la sociedad, haciendo difícil pensar que la vejez se vive de distintas formas dependiendo de la persona y su contexto, que es heterogénea.

En este sentido, el análisis desde la interseccionalidad permite considerar la diversidad de factores que intervienen en esa pluralidad y visibiliza las múltiples formas de opresión estructural que operan en una persona, como la edad, el género, la clase social, la nacionalidad, entre otros, y que, conjugados, operan generando desigualdad y discriminación. "No se puede comprender a la vejez, entonces, como una única, sino como múltiples vejeces, que se van configurando y viviendo situacionalmente dependiendo del contexto y de los sujetos" (Manes et al., 2016, p. 5).

El trabajo con personas mayores, en distintos dispositivos y niveles de prevención, lleva a reflexionar sobre las diversas vejeces, múltiples y heterogéneas, por tanto, el desarrollo de la perspectiva socio-comunitaria de la Terapia Ocupacional, los paradigmas de derechos humanos y psicogerontológico, así como el modelo de apoyo social pueden confluir para abordar el análisis de las problemáticas que surgen a partir de las experiencias de participación ocupacional, las condiciones sociales y culturales en la vejez. Como refieren Carvalho et al. (2008)"el envejecimiento necesita ser pensado y abordado por los terapeutas ocupacionales desde diferentes perspectivas, contemplando también los aspectos sociales que involucran a esta población" (p.31). 
En los últimos años y a partir de los cambios sociales, la Terapia Ocupacional ha trascendido de una práctica individual-clínica hacia una práctica comunitaria-social y contextual, producto de nuevas necesidades de las comunidades, es decir, prácticas de intervención que respondan a cuestiones de naturaleza social (Morrison, 2018). La Terapia Ocupacional, como institución social, está convocada a resolver problemas sociales concretos que involucren a personas y colectivos que se encuentran en condiciones de vulneración, marginalidad y pérdida del bienestar social (Simó et al., 2016).

Esta investigación tuvo como antecedente un estudio correlacional con el cual se propuso estimar la existencia de diferencias significativas respecto de los factores psíquicos protectores, los estereotipos negativos hacia la vejez y el apoyo social en personas mayores que concurren a centros de jubilados o a la sede del Instituto Nacional de Servicios Sociales para Jubilados y Pensionados en Mar del Plata, Argentina. Para ello se compararon dos grupos, el primero estaba integrado por personas mayores que, en calidad de hacedoras, gestionan en las organizaciones; el otro, por aquellas que, en calidad de receptoras, solo concurren a tramitar subsidios. Se hallaron diferencias significativas en las tres variables, con mayor prevalencia de factores psíquicos protectores y apoyo social percibido en las personas mayores hacedoras (Xifra, 2020).

Esa experiencia motivó la necesidad de focalizarse en la población que asiste a comedores comunitarios o recibe alimentos a través del Programa Pro Bienestar del Instituto Nacional de Servicios Sociales para Jubilados y Pensionados.
Se trata de un programa alimentario de impacto comunitario ejecutado por los centros de jubilados, a través del cual se subsidia económicamente a los comedores y se brinda a las personas afiliadas un bolsón que incluye productos secos para complementar la alimentación diaria. Las entrevistas a trabajadoras sociales del Instituto, realizadas en el marco del estudio antes mencionado, permitieron conocer la importancia del programa para personas mayores en condiciones de pobreza. En él tienen prioridad las y los mayores de 70 años y se consideran otros indicadores de vulnerabilidad social para evaluar la condición y la necesidad del beneficio. Los centros de jubilados se constituyen como un dispositivo que, entre otras funciones, habilitan la posibilidad de tejer redes de apoyo y lazos sociales (Xifra, 2020).

Vaux (1990) se refiere al apoyo social como un proceso complejo y dinámico que parte de los intercambios que se dan entre los individuos de una red, en respuesta a las demandas surgidas en ese contexto. En cuanto a la red social, Infante (1990) la define como el conjunto de contactos que brindan a los sujetos identidad social y apoyos de diferentes tipos e impactan en la búsqueda de ayuda. Revilla et al. (2005), por su parte, connotan lo personal y subjetivo asociado con el apoyo social y resaltan el análisis objetivo de la estructura social de la red, es decir, la importancia de considerar aspectos cualitativos y cuantitativos en esta cuestión.

La Organización Mundial de la Salud (OMS, 2002) menciona al apoyo social entre los principales determinantes del envejecimiento activo y lo destaca como uno de los factores cardinales que mejoran la salud, la participación 
y la seguridad. Asimismo, resalta cómo el apoyo social inadecuado se asocia con el aumento de la morbilidad, la mortalidad y el deterioro de la salud. Posteriormente, utiliza el concepto de envejecimiento saludable y refuerza la importancia del entorno y de las relaciones sociales como factores intervinientes en este (OMS, 2019).

Durante el envejecimiento pueden acontecer diversas situaciones como la jubilación, la migración, la pérdida de la pareja, de familiares, amigas o amigos; estas pueden producir disrupciones en la red de apoyo social, reducir su tamaño y su funcionalidad (Arias, 2004). Además, estos acontecimientos también tienden a impactar negativamente en las experiencias ocupacionales de las personas mayores $y$, por tanto, en su adaptación ocupacional (Krzemien et al., 2020); en estos casos es fundamental la construcción de una nueva identidad ocupacional con el compromiso de participación en ocupaciones significativas de manera eficaz y competente (Palma et al., 2019).

Numerosas investigaciones (Fratiglioni et al., 2004; Bishop et al., 2006; Phillips et al., 2008; Vivaldi y Barra, 2012; Castellano, 2014; Carmona, 2015; Alfonso et al., 2016; Garza-Sánchez y González-Tovar, 2018; Glidden et al., 2019) han aportado evidencia sobre el impacto del apoyo social en diversos procesos relacionados con el estado de salud y enfermedad, el bienestar emocional, la calidad de vida, la participación social y, también, la intervención en problemas psicosociales. La presencia de apoyo social es un factor importante a considerar cuando una persona mayor afronta cambios vitales. Disponer de apoyo social es fundamen- tal, no solo por su asociación con el bienestar, también porque en la edad avanzada o ante la irrupción de una enfermedad puede ser necesario contar con soportes para la realización de las actividades de la vida diaria (AVD) y la actividades instrumentales de la vida diaria (AIVD) (Arias et al., 2020).

Investigaciones anteriores llevadas a cabo en Mar del Plata han mostrado que los hijos e hijas, la pareja, los amigos y las amigas ocupan un lugar central en la vejez; a su vez han evidenciado diferencias por género, edad y grupo conviviente en la elección del vínculo que brinda mayor satisfacción (Arias y Polizzi, 2011; Polizzi y Arias, 2014; Arias, 2015; Arias et al., 2015). Si bien no abordan específicamente grupos vulnerables o la consideración del estrato socioeconómico, Polizzi y Arias (2014) señalan la necesidad de realizar investigaciones que aborden poblaciones mayores con desventajas sociales o económicas.

En esta investigación se adopta el término vulnerabilidad para categorizar aquellos grupos de personas privadas de recursos materiales y simbólicos o que se encuentran en situación de marginación, exclusión e inseguridad social (Dimenstein y Neto, 2020). Cecchini y Martínez (2011) describen que el fenómeno del envejecimiento poblacional rápido y creciente en la mayoría de los países de Sudamérica tiene como denominador común que se da en contextos de pobreza y extrema desigualdad, haciendo a las personas mayores especialmente vulnerables. La pobreza y la falta de mecanismos de protección frente a los riesgos de quienes viven en situación de vulnerabilidad deben estar en la agenda de todos los países de América Latina. 
A partir de lo expresado, este estudio se propuso conocer y describir el apoyo social percibido y las variables sociodemográficas en personas mayores que concurren a comedores comunitarios o reciben bolsón de alimentos, registradas en el Programa Pro Bienestar del Instituto Nacional de Servicios Sociales para Jubilados y Pensionados en la ciudad de Mar del Plata.

\section{Metodología}

Desde un enfoque cuantitativo, se llevó a cabo un estudio descriptivo, con un diseño no experimental de corte transversal.

La población estuvo constituida por personas mayores de ambos sexos que concurren a comedores comunitarios $\mathrm{O}$ reciben bolsón de alimentos en centros de jubilados y que están registrados en el Programa Pro Bienestar del Instituto Nacional de Servicios Sociales para Jubilados y Pensionados en la ciudad de Mar del Plata. Se trabajó con un muestreo no probabilístico por conveniencia o accidental (Polit y Hungler, 2000). Los criterios de exclusión incluyeron ser menor de 60 años y tener dificultad para responder los cuestionarios utilizados en la investigación.

Para la recolección de datos se elaboró un cuestionario sociodemográfico que incluye las variables edad, sexo, nacionalidad, nivel de instrucción, estado civil, grupo de convivencia, participación en actividades/talleres en centros de jubilados, participación en actividades recreativas/deportivas en alguna institución o club.

El apoyo social percibido fue medido con el Cuestionario de Apoyo Social (Medical Outcomes Study - MOS) elaborado por Sherbourne y Stewart (1991). Se trata de un instrumento multidimensional que permite estudiar los aspectos cuantitativos (red social) y cualitativos (dimensiones del apoyo social). Se caracteriza por ser breve, de fácil comprensión y autoadministrado. El cuestionario fue adaptado y validado en Argentina (Rodríguez y Enrique, 2010) y otros países latinoamericanos -como Colombia (Londoño et al., 2012)-, en diversas poblaciones y grupos etários. Está compuesto por 20 ítems. El primer ítem hace referencia al tamaño de la red social y los 19 ítems restantes a cuatro dimensiones del apoyo social funcional: emocional informacional, instrumental, interacción social positiva y apoyo afectivo. Con una escala de respuesta de 5 puntos, se pregunta con qué frecuencia está disponible para el sujeto cada tipo de apoyo (escala de Likert). Los valores de corte para cada una de las dimensiones y para la puntuación total del apoyo social percibido se presentan en la tabla 1.

Tabla 1. Valores de corte total y por dimensiones para el Cuestionario de Apoyo Social - MOS

\begin{tabular}{|l|c|c|c|}
\hline Dimensión & Mínimo & Medio & Máximo \\
\hline Apoyo emocional informacional & 8 & 24 & 40 \\
\hline Apoyo instrumental & 4 & 12 & 20 \\
\hline Interacción social positiva & 4 & 12 & 20 \\
\hline Apoyo afectivo & 3 & 9 & 15 \\
\hline Puntuación total del apoyo social percibido & $\mathbf{1 9}$ & $\mathbf{5 7}$ & $\mathbf{9 5}$ \\
\hline
\end{tabular}

Fuente: elaboración propia a partir de Suarez (2011). 
Los cuestionarios fueron aplicados entre el segundo semestre del 2017 y el primer semestre del 2018. Los datos recolectados fueron informatizados y luego

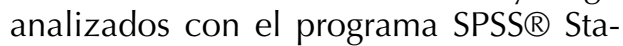
tistics 19. Se obtuvieron estadísticos descriptivos por variables y se aplicó Alfa de Cronbach para valorar la fiabilidad del Cuestionario de Apoyo Social - MOS.

\section{Aspectos éticos}

Para administrar los instrumentos a las personas mayores se solicitó autorización del Instituto Nacional de Servicios Sociales para Jubilados y Pensionados. Las personas participantes fueron contactadas en los centros de jubilados donde funciona el comedor comunitario o se hace entrega del bolsón de alimentos. La recolección de datos se realizó en los comedores Ceram; Reneé Batistesa, y Fe, Esperanza y Dignidad, y en los centros Bernardino Rivadavia, Cerrito San Salvador, Tercera Edad Solidaria y San Cayetano, que hacen entrega de bolsón. Las y los participantes fueron invitados a colaborar con el estudio y a completar de forma voluntaria y auto-administrada los cuestionarios. Se aseguró el anonimato y la confidencialidad en el tratamiento de la información a través de consentimiento informado. En algunos casos las personas mayores solicitaron que se les asistiera, por lo que se administró en el contexto de una entrevista personal.

\section{Resultados}

\section{Caracterización sociodemográfica}

La edad promedio de las personas participantes fue de 74,23 años (s = 8,26); el
$25,71 \%$ de la muestra se encuentra por encima de los 80 años. El 68,6 \% mujeres y el $31,4 \%$ varones. El $40 \%$ son personas beneficiarias que concurren al comedor comunitario; el 60 \% reciben bolsón de alimentos. Respecto a la nacionalidad, en el $97,1 \%$ es argentina.

En cuanto al nivel de instrucción, la mayor parte del grupo presenta primario completo $(31,4 \%)$ e incompleto $(25,7$ $\%)$; el $22,9 \%$ secundario completo y el $8,6 \%$ incompleto; solo un $8,6 \%$ posee universitario incompleto. Mientras que en las mujeres hay un alto porcentaje con estudios primarios incompletos $(29,2$ $\%$ ) y no se registran casos con estudios universitarios, los hombres exhiben el mayor porcentaje con estudios primarios completos $(45,5 \%)$ y presentan casos con universitario incompleto $(9,1 \%)$ y completo $(9,1 \%)$.

La figura 1 permite observar que la mayor parte de la muestra está conformada por personas viudas $(42,9 \%)$. Hay un mayor porcentaje de mujeres casadas $(25 \%)$ con respecto a los varones $(18,2 \%)$. La figura 2 muestra la predominancia de personas mayores que viven solas (54,3\%), siendo esto más frecuente en los varones $(72,7 \%)$.

Respecto a la participación en actividades y talleres propuestos en centros de jubilados, solo un $17,1 \%$ refirió participar, frente al $82,9 \%$ que no lo hace. En esta variable no se observaron diferencias por género. También se indagó sobre la participación en actividades recreativas o deportivas en otras instituciones distintas a los centros de jubilados, hallándose un bajo porcentaje en la categoría participa (20\%), siendo mayor la proporción de mujeres $(20,8 \%)$ que la de varones $(9,1 \%)$. 
Figura 1. Estado civil de personas mayores participantes en el estudio

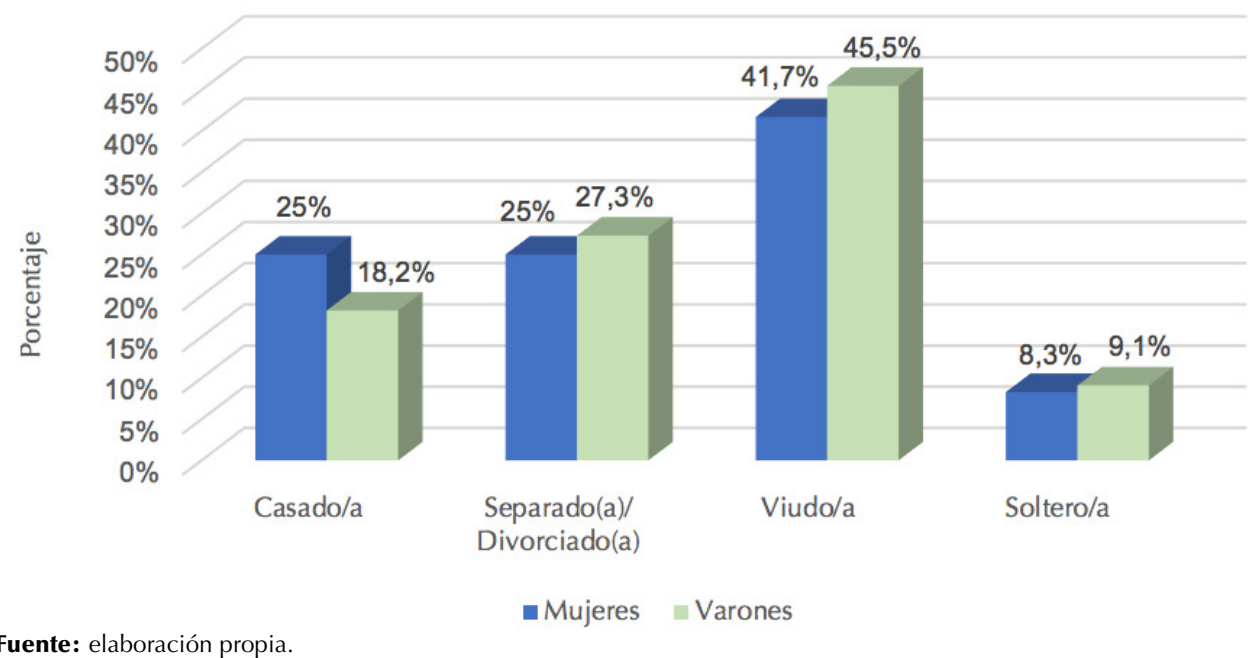

Fuente: elaboración propia.

Figura 2. Convivencia de las personas mayores participantes en el estudio

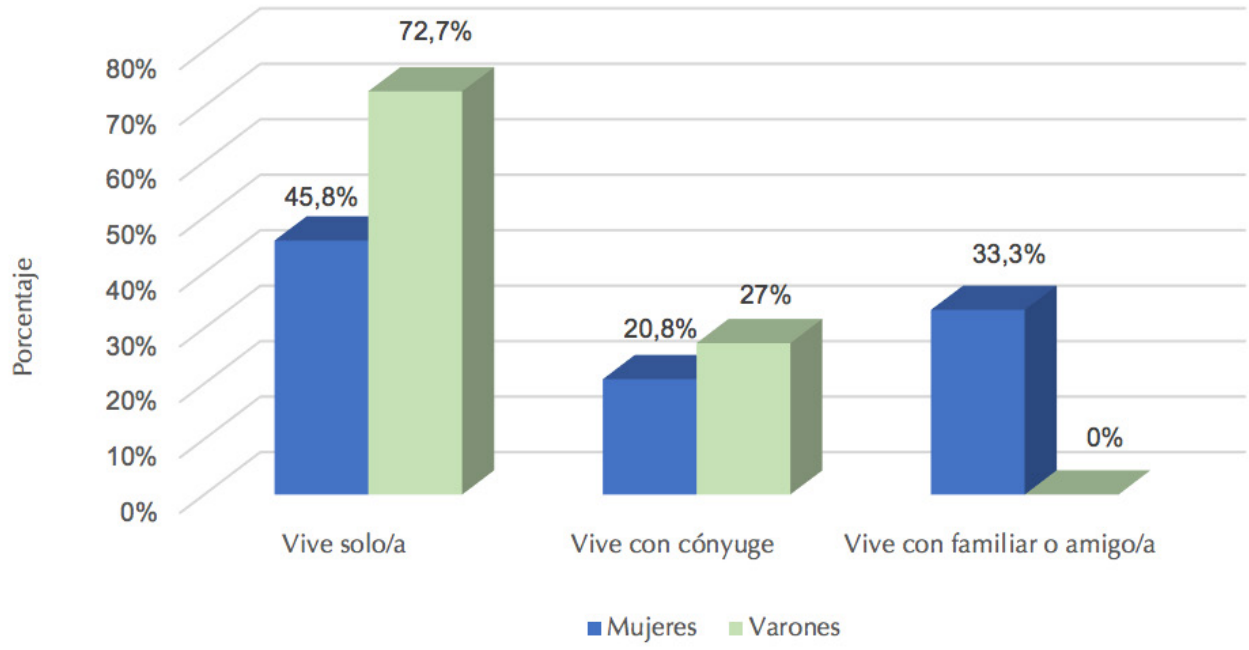

Fuente: elaboración propia.

\section{Apoyo social}

En cuanto al apoyo social percibido (ver tabla 2), cinco personas refirieron no tener ningún amigo o amiga, seis tener solo uno o una y siete tener dos, cons- tituyendo el $51,4 \%$ de la muestra. Respecto a la cantidad de familiares, cuatro refirieron no tener familiares, cinco tener uno o una y tres tener dos familiares, re- 
presentando el 34,3\% de la muestra. Las mujeres presentan una media mayor de familiares $(\mu=8,63)$ y amigos o amigas $(\mu=4,27)$ que los varones $\mu=6,55$ y $\mu=$ 3,37 , respectivamente.

En la tabla 3 se aprecia que las personas mayores presentan una percepción de apoyo social medio, de acuerdo con los puntajes obtenidos y los valores de corte propuestos (Suarez, 2011). No se hallaron diferencias por género.
Las dimensiones interacción social positiva y apoyo afectivo (tabla 3), definidas a partir de las posibilidades autopercibidas de disponer de alguien con quien compartir momentos gratificantes y recibir demostraciones reales de amor, cariño o empatía (Revilla et al., 2005), exhibieron resultados superiores a los valores medios de corte.

Tabla 2. Cantidad de amigos y familiares (media, desviación estándar y valor mínimo y máximo)

\begin{tabular}{|l|c|c|}
\hline Valores & $\begin{array}{c}\text { Cantidad de } \\
\text { amigos/as }\end{array}$ & $\begin{array}{c}\text { Cantidad de } \\
\text { familiares }\end{array}$ \\
\hline Media $(\mu)$ & 3,63 & 7,97 \\
\hline Desviación estándar $(s)$ & 3,97 & 7,50 \\
\hline Mínimo & 0 & 0 \\
\hline Máximo & 20 & 30 \\
\hline
\end{tabular}

Fuente: elaboración propia.

Tabla 3. Valores obtenidos en apoyo social percibido total y en sus dimensiones

\begin{tabular}{|l|c|c|c|c|c|}
\hline Dimensiones & N & Mínimo & Máximo & Media $(\mu)$ & Desviación (s) \\
\hline Apoyo social percibido total & 35 & 20 & 95 & 62,77 & 17,11 \\
\hline Apoyo emocional informacional & 35 & 9 & 40 & 25,46 & 7,96 \\
\hline Apoyo instrumental & 35 & 4 & 20 & 13,46 & 4,94 \\
\hline Interacción social positiva & 35 & 4 & 20 & 12,46 & 3,54 \\
\hline Apoyo afectivo & 35 & 3 & 15 & 11,40 & 3,38 \\
\hline
\end{tabular}

Fuente: elaboración propia.

Si bien el apoyo instrumental, es decir, la provisión de ayuda material perceptible que pueda recibir la persona, y el apoyo emocional informacional, entendido como expresión de afecto, comprensión empática, guía, oferta de consejo e información (Revilla et al., 2005), también presentaron valores medios superiores a los puntos de corte, se registraron casos de personas mayores que expresaron nunca o pocas veces disponer de alguien que les prepare un plato de comida (10), les ayude en tareas domésticas (10), les asista si tuvieran que quedarse en cama (8), contar con alguien cuando necesitan hablar (11) o compartir sus temores y problemas más íntimos (13). 
Por último, se registró un alto grado de fiabilidad en el Cuestionario de Apoyo Social - MOS $(a=0,81)$.

\section{Discusión}

Esta investigación se propuso aportar conocimiento sobre las personas mayores que concurren a comedores o son beneficiarias del bolsón de alimentos. El Programa Pro Bienestar busca tener impacto comunitario, satisfacer necesidades básicas de alimentación, pero también habilitar un espacio de compañía -en el caso del comedor-, entendiendo que es una de las necesidades de las personas que vive en condiciones de soledad.

Las características sociodemográficas prevalentes en las personas mayores participantes en este estudio han sido la presencia de un bajo nivel de instrucción, un elevado porcentaje de viudos y de personas que viven solas, a lo que se suma la escasa participación en actividades o talleres educativos, recreativos, etc. Los resultados en cuanto a escolaridad, estado civil y convivencia coinciden con los hallazgos de Arias y Polizzi (2011), Arias et al. (2015), Arias et. al. (2020) y Xifra (2020) respecto a adultos mayores residentes en la ciudad de Mar del Plata.

A través del Cuestionario de Apoyo Social - MOS se pudo hacer una aproximación al conocimiento sobre la percepción de apoyo social desde una perspectiva estructural y funcional. En cuanto a la cantidad de amigos/amigas y familiares, las personas mayores participantes en esta investigación han presentado un promedio menor que aquellas involucradas en actividades de gestión en centros de jubilados (Xifra, 2020). Es pertinente mencionar que un alto porcentaje carece de estos lazos o tiene solo una o dos relaciones de este tipo.

De manera consistente con estudios anteriores (Polizzi y Arias, 2014; Arias, 2015) que evidenciaron que en su mayoría las redes de apoyo social evaluadas presentaban carencias y no cumplían con sus funciones, este estudio reveló que buena parte de las personas mayores participantes cuenta con poca o ninguna frecuencia con alguien que les brinde apoyo instrumental y emocional informacional.

Si bien los puntajes obtenidos al aplicar y analizar el Cuestionario de Apoyo Social - MOS registran valores medios respecto a los puntos de corte propuestos (Suarez, 2011), el apoyo social percibido total y en las cuatro dimensiones es menor que en la población de personas mayores que gestionan en los centros de jubilados, es decir, las hacedoras (Xifra, 2020).

En aquellos estudios donde las personas mayores participantes (Alfonso et. al., 2016) tienen mayor nivel de instrucción y mayor porcentaje de casados, o características sociodemográficas similares pero asisten a centros de día y presentan alta participación en actividades (CasteIlano, 2014), estas han mostrado un mayor apoyo social en comparación con el grupo de esta investigación. Finalmente, respecto a estudios que hayan considerado el estrato socioeconómico (Bishop et.al., 2006), se ha detectado que esta variable se asocia con la percepción de apoyo social y ambas tienen un efecto directo sobre la salud. 
La red de apoyo social tiene las características de un sistema abierto, encontrándose en un proceso de construcción y reconstrucción permanente. En la vida adulta esta suele ser heterogénea y amplia, debido fundamentalmente al desempeño de múltiples roles que la enriquecen; en la vejez, por diversas razones, algunos lazos se van perdiendo, la red tiende a sufrir disrupciones, reducir su tamaño y perder funcionalidad. Así, algunas situaciones que pueden generar cambios en la red durante la vejez son la jubilación, la pérdida de familiares y amigos o amigas, la pérdida de la pareja y la migración (Arias, 2004).

Estos acontecimientos también pueden afectar el desempeño ocupacional y producir desequilibrios. Por tanto, la adaptación ocupacional, entendida como la capacidad de construir una identidad ocupacional positiva, se desarrolla como reacción ante situaciones complejas, pero también como la habilidad cotidiana para progresar en las opciones ocupacionales y mejorar el bienestar (Palma et al., 2019). En la población de estudio, además de los acontecimientos mencionados, esperables o no en esta etapa vital, se suma la vulnerabilidad social derivada de la ausencia o la escasez de recursos económicos. En este contexto la búsqueda debe apuntar a que los sujetos puedan construir una nueva identidad ocupacional a pesar de las situaciones adversas, con la articulación de sus propios intereses ocupacionales y las posibilidades del entorno.

El análisis desde la interseccionalidad permite considerar los factores estudiados e identificados que operan generando desigualdad (Manes et al., 2016). La edad, el género, el nivel de instrucción y la clase social de las personas mayores que concurren a los comedores o reciben bolsón conduce a reflexionar sobre las múltiples dificultades para acceder de forma igualitaria a los recursos sociales. Se trata de una adición de condiciones o factores que potencian los riesgos u obstaculizan las posibilidades de desarrollo. La interacción entre distintos vectores, como en este caso la edad, el acceso que hayan tenido a la educación, las diferencias de género y los recursos económicos, ha impactado de diversas formas facilitando situaciones y experiencias únicas (Manes et al., 2016).

Finalmente, aunque los centros de jubilados son organizaciones sin fines de lucro que se constituyen como dispositivos comunitarios, ofrecen múltiples opciones (actividades de movimiento, artísticas, psicoeducativas, cognitivas, etc.) y a su vez construyen, remarcan y desarrollan el sentido de pertenencia social en las personas mayores, es importante resaltar la baja participación en talleres propuestos en estos centros, así como en actividades recreativas o deportivas en otras instituciones. Estudios anteriores han destacado que, a pesar de la pérdida de vínculos familiares y sociales en la vejez, las personas mayores incorporan nuevos lazos que permiten compensar y mantener el tamaño de sus redes (Arias y Polizzi, 2013).

En este sentido, desde una visión amplia y social de la Terapia Ocupacional (Simó et al., 2016), pensar en propuestas socializadoras que faciliten captar el interés y la motivación en las personas mayores a participar en estos espacios puede favorecer su acceso a ocupaciones significativas y repercutir en el bienestar y la posibilidad de establecer nuevas relaciones y vínculos de apoyo. 


\section{Conclusiones}

Si bien la cantidad de vínculos de que disponen las personas mayores concurrentes a comedores o que reciben bolsón en el Programa Pro Bienestar no es escasa ni se evidencian disrupciones severas, coexisten una menor disponibilidad de apoyos, en particular en las dimensiones instrumental y emocional informacional, un porcentaje alto de personas mayores que viven en soledad, el bajo nivel de instrucción y una escasa participación en centros de jubilados.

Comprender la realidad social y comunitaria, la disponibilidad de apoyos y las posibilidades de participación en dispositivos comunitarios de estos colectivos de personas mayores visibiliza problemáticas particulares. Así, la irrupción de alguna patología que afecte el desempeño en las actividades de la vida diaria o en las actividades instrumentales de la vida diaria muy probablemente pondrá en evidencia la escasez de apoyos, en particular instrumental. En esas circunstancias, la necesidad de cuidados, la dependencia, la morbilidad y la institucionalización se convierten en punto central de una misma problemática atravesada por las condiciones de vulnerabilidad.

La falta de recursos materiales y económicos, junto con condiciones negativas del entorno pueden estar relacionadas con las posibilidades de participación y el compromiso en actividades significativas. La participación de manera activa, autónoma y el desempeño en ocupaciones significativas podrían favorecer la construcción de una nueva identidad ocupacional y la continuidad del proyecto vital, ayudar a contrarrestar la soledad y el ais- lamiento, y proveer oportunidades de inclusión y participación.

Las y los terapistas ocupacionales, como profesionales de la salud que se desempeñan en diversos dispositivos socio-comunitarios, deben poner en práctica estrategias de prevención y promoción de la salud y diseñar intervenciones que habiliten la participación de las personas mayores en espacios y ocupaciones significativas, en una práctica social centrada en superar las injusticias, las desigualdades y la falta de oportunidades.

Si se entiende que envejecer formaría parte de una construcción colectiva (Silva et al., 2010), debería apuntarse a un mundo con más personas mayores en la vida social, cultural, económica y política. Cuando, además, estos sujetos se encuentran en condiciones de vulnerabilidad, deben ofrecerse respuestas estructurales que brinden oportunidades sociales e involucren a profesionales, autoridades públicas, organizaciones y movimientos sociales.

Una limitación de este estudio fue el tamaño y la distribución por género de la muestra. Con una muestra mayor, sería ideal realizar comparaciones de medias y correlaciones de variables. Se propone realizar estudios complementarios, establecer cuotas por género o condición de beneficio (comedor, bolsón), así como una triangulación metodológica que, desde una perspectiva cualitativa, profundice sobre la trayectoria vital de las personas mayores teniendo en cuenta aspectos individuales y contextuales. 


\section{Referencias}

Alfonso, L., Soto, D. y Santos, A. (2016). Calidad de vida y apoyo social percibido en adultos mayores. Revista de Ciencias Médicas de Pinar del Río, 20(1). https://www.medigraphic. com/pdfs/pinar/rcm-2016/rcm161l.pdf

Arias, C. (2004). Red de apoyo social y bienestar psicológico en personas de edad. Ediciones Suarez.

Arias, C. (2015). Red de apoyo social y bienestar psicológico en personas mayores [tesis de maestría, Universidad Nacional de Mar del Plata]. Repositorio RPSico. http://rpsico.mdp. edu.ar/handle/123456789/402

Arias, C. y Polizzi, L. (2011). La relación de pareja. Funciones de apoyo y sexualidad en la vejez. Revista Temática Kairós Gerontología, 14(10), 49-71. https://doi.org/10.23925/2176-901X. 2011v14iEspecial10p49-71

Arias, C. y Polizzi, L. (2013). El apoyo social en la vejez: la familia, los amigos y la comunidad. Revista Kairós Gerontología, 16(6), 25-40. https://doi.org/10.23925/2176-901X. 2013v16i4p313-329

Arias, C., Polizzi, L y Pantusa, J. (2015). Vínculos que brindan apoyo social a las personas mayores. Diferencias por género. En Memorias del VII Congreso Internacional de Investigación y Práctica Profesional en Psicología XII Jornadas de Investigación (pp. 7-12). Facultad de Psicología, Universidad de Buenos Aires. https://www.aacademica.org/000-015/34

Arias, C., Bozzi, N. y Soliverez, C. (2020). Tamaño, composición y funcionalidad de la red de apoyo social en la vejez avanzada (vol 1). En E. Coelho Sampaio (ed), Envelhecimento Humano. Desafíos Contemporaneos (pp. 642-654 ). Editora Científica Digital. https:// doi.org/10.37885/200901511
Bishop, A., Martin, P. y Poon, L. (2006). Happiness and congruence in older adulthood: astructural model of life satisfaction. Aging Ment Health, 10(5), 445-53. https://doi. org/10.1080/13607860600638388

Carmona, S. (2015). La contribución de la vida social al bienestar en la vejez. Entreciencias, 3(8), 393-401. http://www.redalyc.org/articu$\underline{\text { lo.oa? id }=457644946010}$

Carvalho, C., Malfitano, A. y Lopes, R. (2018). Intervenciones sociales de terapia ocupacional con adultos mayores en América del Sur: una revisión no sistemática de la literatura. Revista Chilena de Terapia Ocupacional, 18(2), 2943. https://revistaatemus.uchile.cl/index.php/ RTO/article/view/48822

Castellano, C. (2014). La influencia del apoyo social en el estado emocional y las actitudes hacia la vejez y el envejecimiento en una muestra de ancianos. International Journal of Psychology and Psychological Therapy, 14(3), 365-377.

Cecchini, S. y Martínez, R. (2011). Protección social inclusiva en America Latina: una mirada integral, un enfoque de derechos. CEPAL.

Cicciari, M.R. (2017). Informe sobre las condiciones de la vida de las personas mayores en la ciudad de Mar del Plata. Pontificia Universidad Católica Argentina.

Dimenstein, M. y Neto, M.C.(2020). Enfoques conceptuales de la vulnerabilidad en el ámbito de la salud y la asistencia social. Pesquisas e Práticas Psicossociais, 15(1), 1-17. https://pesquisa.bvsalud.org/portal/resource/ es/biblio-1098423 
Fratiglioni, L., Paillard-Borg, S. y Winblad. (2004). An active and socially integrated lifestyle in late life might protect against dementia. The Lancet Neurology, 3(6), 343-53. https://doi. org/10.1016/S1474-4422(04)00767-7

Garza-Sánchez. , R. y González-Tovar, J. (2018). El apoyo social en la vejez: diferencias por sexo en una muestra del norte de México. Interacciones, 4(3), 191-198. https://www. revistainteracciones.com/index.php/rin/ article/view/65

Glidden, R., Borges, C., Pianezer, A. y Martins, J. (2019). A participação de idosos em grupos de terceira idade e sua relação com satisfação com suporte social e otimismo. Boletim - Academia Paulista de Psicologia, 39(27), 261-275. http://pepsic. bvsalud.org/scielo.php?script=sci arttext\&pi$\mathrm{d}=\mathrm{S} 1415-711 \times 2019000200011 \& \operatorname{lng}=\mathrm{pt} \& \mathrm{t}-$ $\underline{\text { lng }=p t}$

Huenchuan, S. (2018). Envejecimiento, personas mayores y agenda 2030 para el desarrollo sostenible. Naciones Unidas CEPAL.

Infante, C. (1990). Utilización de servicios de atención prenatal: influencia de la morbilidad percibida y de las redes sociales de ayuda. Salud Pública Mexico, 32(4), 419-429. https://saludpublica.mx/index.php/spm/article/ view/5311

Instituto Nacional de Estadística y Censos [INDEC] (2010). Resultados censo nacional de población, hogar y viviendas 2010. Argentina.

Krzemien, D., Miranda, C. y Martín, C. (2020). La ocupación en la gerontología: una perspectiva crítica del Modelo de Ocupación Humana. Revista Ocupación Humana, 20(1), 82105. https://doi.org/10.25214/25907816.268
Londoño, N.E., Rogers, H., Posada, S., Ochoa, N., Castilla , J.F., Jaramillo, J. y Aguirre, E. (2012). Validación en Colombia del cuestionario MOS de apoyo social. International Journal of Psychological Research, 5(1), 142-150.

Manes, R., Carballo, B., Cejas, R., Machado, E., Prins, S., Savino, D. y Wood, S. (2016). Vejeces desiguales. Un análisis desde el enfoque de los derechos de las personas mayores. Margen (83), 1-13. https://www.margen.org/ suscri/margen83/manes83.pdf

Morrison, R. (2018). ¿Qué une a la Terapia Ocupacional? Paradigmas y perspectivas ontológicas de la ocupación humana. Revista Interinstitucional Brasilera de Terapia Ocupacional, 2(1), 182-203. https://revistas.ufrj.br/ index.php/ribto/article/view/12699

Organización Mundial de la Salud [OMS] (2002). Envejecimiento activo: un marco político. Revista Española de Geriatria y Gerontología, 37(S2), 74-105.

Organización Mundial de la Salud [OMS] (2019). Decada del envejecimiento saludable. OMS. https://cdn.who.int/media/docs/ default-source/decade-of-healthy-ageing/ final-decade-proposal/decade-proposal-final-apr2020-es.pdf?sfvrsn=73137ef 4

Organización de las Naciones Unidas [ONU] (2019). 2019 Revision of World Population Prospects. ONU. https://population. un.org/wpp/

Palma, O., Hueso, E., Martí, C., Fernández , M., Campos, C. y Montoya, R. (2019). Understanding the occupational adaptation process and well-being of older adults in Magallanes (Chile): A qualitative study. International Journal of Environmental Research and Public Health, 16(19), 36-40. http://doi.org/10.3390/ ijerph16193640 
Phillips, D. R., Sui, O.L., Yeh, A. G., y Cheng, K. H. (2008). Informal social support and older person's psychological wellbeing in Hong Kong. Journal of Cross- Cultural Gerontology, 23(1), 39-55. https://doi.org/10.1007/s10823$\underline{007-9056-0}$

Polit, D.F. y Hungler, B.P. (2000). Investigación científica en ciencias de la salud. Mc Graw Hill.

Polizzi, L. y Arias, C. (2014). Los vínculos que brindan mas satisfacción en la red de apoyo social de los adultos mayores. Pensando Psicología, 10(17), 61-70. http://dx.doi. org/10.16925/pe.v10i17.784

Revilla, L., Luna, J., Bailon, E. y Medina, I. (2005). Validación del cuestionario MOS de apoyo social en atención primaria. Medicina de Familia, 6(1), 10-18.

Rodríguez, S. y Enrique, H. (2010). Validación argentina del cuestionario MOS de apoyo social percibido. Psicodebate, 7, 155-168. http://dx.doi.org/10.18682/pd.v7i0.433

Sherbourne, C.D. y Stewart, A.L. (1991). The MOS social support survey. Social Science \& Medicine, 32(6), 705-714. https://doi.org/10.1016/0277-9536(91)90150-B

Silva, H., Lima, A. y Galhardoni, R. (2010). Envelhecimento bem-sucedido e vulnerabilidade em saúde: aproximações e perspectivas. Interface-Comunicação, Saude, Educação, 14(35), 867-877. https://doi.org/10.1590/ S1414-32832010005000034

Simó, S., Guajardo, A., Oliver, F.O., Galheigo, S. y García, S. (eds.) (2016). Terapias ocupacionales desde el sur: derechos humanos, ciudadanía y participación. USACH.
Suarez, M.A. (2011). Identificación y utilidad de las herramientas para evaluar el apoyo social al paciente y el cuidador informal. Revista Médica La Paz, 17(1), 60-67. http://www. scielo.org.bo/scielo.php?script=sci arttext\&pid=S1726-89582011000100010

Vaux, A. (1990). An ecological approach to understanding and facilitating social support. Journal of Social and Personal Relationships, 7, 507-518. https://doi. org/10.1177/0265407590074007

Vivaldi, F. y Barra, E. (2012). Bienestar psicológico, apoyo social percibidoy percepción de salud en adultos mayores. Terapia psicológica, 30(2), 23-29. https://dx.doi.org/10.4067/ $\underline{\text { S0718-48082012000200002 }}$

Xifra, M.J. (2020). Estereotipos, factores psíquicos protectores y apoyo social percibido en adultos mayores hacedores y receptores de la ciudad de Mar del Plata. Revista Argentina de Terapia Ocupacional, 6(2), 42-52. https:// www.terapia-ocupacional.org.ar/revista/RATO/2020jul-art5.pdf 